\title{
USING AGENT BASED MODELING TO INTERPRET UNDERLYING FACTORS OF UNDERREPRESENTATION OF MINORITIES IN HOLLYWOOD FILMS
}

\author{
Carmen A. Iasiello \\ Department of Computational and Data Sciences \\ George Mason University \\ 4400 University Drive \\ Fairfax,VA, USA \\ ciasiell@gmu.edu
}

\begin{abstract}
Based on examination of top grossing films it is clear there exists racial underrepresentation in Hollywood movies. I propose a socioeconomic approach to examine the Hollywood labor system as a network using an agent based model (ABM) within a notional movie labor market based on preferential attachment and compare the findings with 50 co-production ego networks during the 2015 movie cycle. Using an ABM, I test the hypothesis that slight individual preference for racial and ethnic similarity within one's own network at the microlevel sufficiently explains the phenomena of Hollywood underrepresentation at the macrolevel. Using regression analysis of the real world 2015 co-actor networks I test the hypothesis that race/ethnic minority status affects ones position within the network of successful actors. In both cases, the hypotheses are not sufficient to explain the phenomena and I propose further exploration into causes of opportunity loss in accessing the labor market.
\end{abstract}

Keywords: agent based model, social network analysis, racial inequality, labor markets, computational sociology.

\section{INTRODUCTION}

Motion pictures, when considered a form of cultural production as defined by Bourdieu (1993), are the result of their creators' intentions and perspective. As such, how those intentions and perspectives are formed plays a role in the resulting product. Movies are the product of groups of individuals that form the financing, creative production, and performance organization for each production. The form and function of the social networks for these organizations, according to network theorists informs the patterns in these structures (Wasserman and Faust 1994, Kadushin 2012). For movies in Hollywood, there can be considered two marks of success, financial success and awards for the quality of the work. Based on evidentiary work of the movie industry and top grossing films (Bunche Center 2016, Smith et al 2016) and the recent makeup of Oscar nominees in 2015-2016 there exists racial underrepresentation in Hollywood movies.

While Hollywood's consumers are increasingly global (Walls and McKenzie 2012), the creative talent used in major motion pictures from Hollywood are predominantly from the United States. As such, we can take as a comparison the U.S. population which is $67 \%$ white, non-Hispanic, $37 \%$ race and ethnic minority (US Census 2014). Minority actors made up $26 \%$ and $27 \%$ of the actors in the top 700 grossing films in 2013 and 2014, respectively (Bunche Center, 2016). Looking at only lead roles we find that minorities made up $16 \%$ of lead actor roles in the 174 films examined for the annual diversity report on Hollywood published by the Bunche Center (2016). 
This underrepresentation in Hollywood is also present in other fields such as health, law, social science, and others (Lam 2015, Sullivan Commission 2004). In order to determine plausible courses of action for addressing underrepresentation, we must first formulate explanations for the phenomena. In sociology, Bonilla-Silva $(1997,2006)$ argued for a theory of racialization as a means to understand the phenomena as a structural one. However, identifying that structural racism exists does not provide specific courses of action for the studios or actors in Hollywood to consider. I propose a socioeconomic approach to examine the Hollywood labor system as a network.

Unlike many other professions, the labor market for creative talent operates on a system of closed networks with very little oversight outside of public opinion (Smith et al 2013). The role of creative talent in this market Baker and Faulkner (1991) argue is a resource derived from structural, interactionist, and network approaches to the sociological role. As such, can the basic parameters of this market combined with how individual choice operates within it explain the current state of underrepresentation? Schelling $(1971,1978)$ demonstrated computationally that benign individual preferences lead to significant system-wide inequalities. These preferences translate into homophily within one's own network (McPherson et al, 2001). However, they are compounded by structural segregation along racial lines (Massey and Denton 1993). McDonald (2011) examined how these two elements, homophily and structural segregation, restrict or provide access to social resources along gender and racial lines. His work concluded that how resources are accessible or not based on race was dependent on the types of resource.

Using statistical analysis of movie releases and results, DeVany (2004) demonstrated that movie box-office success exists on a Pereto distribution with infinite variability prior to a movie's release, rendering prerelease forecasts unusable. Previous agent based models of the movie market have addressed this phenomenon, Russo's ChatterMod ABM replicates the complexity theorized by DeVany for movies (Russo, 2016). However, previous ABMs have not addressed how actor opportunity based on actor networks or impacts of race on such dynamics.

In this paper, I test the hypothesis: individual preference for racial and ethnic similarity within one's own network at the microlevel sufficiently explains the phenomena of Hollywood underrepresentation at the macrolevel. In order to create the necessary individual level heterogeneity of the persons in the labor market and to allow for success or failure of an individual's prestige in the market, I use agent based modeling as the approach to test this hypothesis. Use of agent based modeling also allows continued examination of additional hypotheses based on the results of this first hypothesis test. To further identify if real world conditions exhibit the microlevel behavior of individual preferences in co-production networks, I explore the second hypothesis: race/ethnic minority status influences one's position within the coproduction networks of successful actors.

In the following paper, I first present, in Section 2, the methodology for examining this topic through both agent based modelling and SNA. In Section 3 I present the findings of the ABM experiments and compare them with the SNA of a real world film actor network. In Section 4, I conclude with possible future work to examine methods of influencing change in areas where significant denial of opportunity along racial lines are significant.

\section{METHODOLOGY}

The research question driving this exploration of both hypotheses is "Does individual preference for racial and ethnic similarity within one's own network sufficiently explain the phenomena of Hollywood underrepresentation?" To explore this question requires both a simulated environment within which to tests levels of this particular behavior. It also requires exploration of real world networks and the structures inherent in working actors' coproduction. As such I explore this question in two phases. First, I examine mechanisms of network creation in a movie labor market using an ABM. Then, I examine real world actor networks through social network analysis methods. 


\subsection{ABM Overview, Design Concept, and Details}

Previous agent based modelling work has shown that network dynamics impact gendered hiring (Tassier 2008 ) and that identity-based segregation impacts the institutional environment for group production (Rhee and Kim 2014). Additionally, the networks of movie production have been explored to show the impact of specific groups of actors (Herr et al 2007). The experiments discussed here use an agent based model (ABM) conducted in Netlogo. The ABM includes heterogeneous agents connected via a random network that establishes its form based on individual preference for similarity within one's own network.

Additionally, given that the labor market for Hollywood is highly dependent on the success of one's past efforts (Smith et al 2016), the network also forms along preferential attachment to connections who have a higher status than oneself. This behavior takes place in a movie labor market space to replicate status derived from movie success (or lack thereof).

To test the first hypothesis, the model is calibrated to be populated with a percentage of minority agents available to the market that mirrors that of the United States: $37 \%$. I similarly calibrate the initial movie makers within the space to match the racial makeup of those in the real world major motion picture studios: near 100\% white, non-Hispanic (Bunche Center 2016). To establish whether the results conform to the hypothesis or disprove it, the results are compared to a network I composed of the actors in the top ten movies of 2015 and their networks of actors based on the other 2015 movies they were cast. For this comparison data, I drew the actor information from the Internet Movie Database (IMDB). At the macro level I will compare the percentage of minorities in the movie space of the ABM (discussed below) with the percentage of minorities in both the Smith et al study and the independently devised 2015 top movies actor network.

It is important in any scientific experimentation to ensure the ability to replicate the findings when replicating the experiment. In the field of computational social science, it is not uncommon for the experimentation to be largely or wholly done within an ABM. In order to facilitate the replication of the ABM experiments, Grimm et al argue for the use of an Overview, Design Concept, and Details (ODD) protocol to allow for a common language of implementation regardless of computer programing language for the ABM itself. To this end, while I've developed the ABM and conducted the experiments in Netlogo, I use the ODD protocol to explain the ABM so it may be replicated elsewhere in another computing language or platform.

\subsubsection{Abstract spatial components - The "Movie Market"}

The cell space is a 33 by 33 grid and represents the film creative labor market. According to Sociology of Work, within this specific labor market, "the most tangible signal of future productivity is a worker's association with prior successful projects." While the labor pool of the film industry was once determined by an actor's association with a studio, this is no longer the case. The firm plays little role in this form of labor market and instead the labor is parsed by reputation and talent agencies. As such, "Hollywood's labor market is highly segmented, in part because of the opportunity structures created by the network of relations of talent agencies...In the absence of personnel systems that hold decision makers responsible for unbiased decisions, those in charge rely instead on closed social networks of interpersonal ties and informal subjective criteria for evaluating creative workers' contributions (Smith 2013)." Rather than instantiating firms, I chose to have the agents interact on a market space.

In order to replicate such a labor market, the cell space consists of a "movie space" and a "non-movie space.". The movie space is centrally located and consists of 356 cells with an associated score between 0 and 1. The success of a project is represented by the higher the score. For the purposes of the model, I assume the success of a movie is random. This assumption is consistent with current theory and existing evidence that the success of a movie cannot be predicted by the inputs themselves but rather is determined via a complex interplay of the inputs and the network of movie goers (De Vany 2004, Russo 2016). The scores are distributed among the cells stochastically at each time step. The non-movie space is the 733 cells 
surrounding the movie space. Here, there are no associated scores associated with the cells. This space serves as a holding pattern for agents who could be employed in a movie, but currently are not.

\subsubsection{Agent Attributes}

\section{Race/Ethnicity:}

In order to examine the crux of my research question, the agents are embedded with a binary racial/ethnic attribute. The agents are either a race/ethnic minority or they are white non-Hispanic. The representation of different races and ethnicities in Hollywood movies is inherently part of a feedback loop about race, culture, and stereotypes. Two ways to explore this phenomenon would be 1) examining the underrepresentation of minorities and 2) examining the overrepresentation of white, non-Hispanics. While similar, they are not the same. All racial minorities are currently underrepresented in films. However, they are not underrepresented to the same degree. For example, the question of underrepresentation would include levels at which one minority may be underrepresented to a greater degree than another minority. I focus the model on overrepresentation of white, non-hispanics to first examine the simplest possible explanation for the phenomena overall.

Within the model, the agent's minority status is scripted as either true or false and visually displayed as a blue or red colored agent. The minority status is the engine for the similarity of network calculations done by the main algorithm tested. Similar to other segregation models such as the Schelling Segregation model by Wilensky (1997) in Netlogo and Moujahid (2014) in Python, the agents here are satisfied when a level of similarity is met. Here, the agents determine their satisfaction by whether the composite of the agents with whom they are directly linked conforms to the percentage desired for similarity. If it does not, they break links with individuals that do not also have their minority status.

\section{Level of Success:}

Success for the agents is measured by score. The score is derived from interaction between the agent and the movie space.

Status as "in movies" and "on screen":

Actors are cast in movies and once cast they are marked as "on screen." In addition, to capture the role of talent agents and others who are not on screen in distributing labor, a predetermined number of agents are encoded as in movies, but not necessarily as creative talent. This marker at instantiation allows agents to be pulled into the movie space at the beginning of the simulation based on a network. Because agents encoded with this marker represent individuals who may be talent agents, producers, or others who play a role in the movie production, they are able to reap a score from a cell similar to the agents encoded solely as actors and for the purposes of testing behave the same as the actor agents. They are separated here to allow for future work that would require the demarcation.

\subsection{Initialization}

At the initialization of the model, agents are assigned a permanent race minority status based on the percentage set by the experimenter. For the experiments reported here, race minority status is true for about 37 percent of agents. Agents are assigned a permanent gender based on a uniform distribution. The number of agents that are initially "in-movies" (e.g. producers, casting directors) is set by the experimenter as a percentage of the agents based on minority status. This allows the experimenter to set the parameters such that racial makeup of the initial movie executives bringing in talent matches that found empirically. The agents that are encoded with this attribute remains the same for the entire run.

The initial number of agents that are working actors or "on-screen" may be adjusted by the experimenter. For the experiments reported here, I have set this to $10 \%$ of agents in order to allow for an initial population 
of actors making movies. As the simulation runs, whether an agent is encoded with this attribute changes based on whether the agent is actively making movies.

The attributes of "in-movies" and "on-screen" are not mutually exclusive and a single agent may be both. This mimics real world dynamics where an actor may also be a producer. And for the purposes of the model ensures all the agents in the simulation are able to become working actors for the purposes of testing the research question.

All agents receive at least a level of success or score of 0.5 . There is an initial 0.1 addition to an agents score for each of the "in-movies" or "on-screen" attributes. As such, the agents initially have a score between 0.5 and 0.7 . The floor for the score throughout the run of the model is 0.1 .

If an agent is encoded as a race minority, they are encoded with a potential for opportunity loss set by the experimenter. For example, if the experimenter sets the opportunity loss at 0 , the model assumes no difference in opportunity among agents based on race. If the experimenter sets the opportunity loss at $50 \%$, about half the agents encoded as a race minority will have the potential to lose their "on-screen" status. This potential is activated in one variation of the testing parameters.

All the agents are initially situated on the "non-movie space" randomly. They are then connected in a spatially clustered network based on the network initialization of Stonedahl and Wilensky's Virus on a Network Netlogo Model. An agent is randomly chosen and connected to the nearest agent to which it is not already connected. This process repeats until the average node degree specified by the experimenter is reached (Stonedahl and Wilensky 2008).

\subsubsection{Baseline Activation Schedule}

At each time step, the agents lose a small amount of score $(0.1)$ to represent the currency of reputation economics in the actor labor market. The floor for the score is 0 , no agent can have a negative score. The agents encoded with "in-movies" or "on-screen" then move to the movie space. The movie space cells are then assigned the successfulness score for the movie, termed "movie points." For the purposes of the model, movie points are set as a random float between 0 and 1 . Success of those "in-movies" and "on-screen" is then calculated and the movie points are then added to the scores of the agents situated on that cell.

Next, the agents assess whether they are satisfied with their immediate network in terms of percentage of connections with a similar race minority status to themselves. If the percentage of their directly linked contacts is greater than the percentage required for satisfaction as set by the experimenter, they do nothing. If it is not, they seek out a new agent to with whom to link. The agents then experience a casting call process. Now, that their networks may have changed, they examine whether they are connected to someone "in movies." If they are, they're status of "on-screen" changes to true. A level of noise may be set in the model by the experimenter. Based on the level set (between 0 and 5\%) by the experimenter, a small percentage of the agents experience a "change of luck." For this small percentage of agents, if they are currently "on screen" and have at least one link with another neighbor in that network, they experience "bad luck" whereby they lose one connection, their "on screen" status, half of their score, and are moved to the "nonmovie space." If they are not already "on screen," they gain "on screen" status and are prepped to launch into the movie space on the next time step.

Status Seeking Linking:

For baseline experimentation, there is an option to allow the network to link via a preferential attachment process. Here, once a tie is removed the agents choose with whom to randomly link next based on score. They seek out an agent with a level of success higher than their own and link with them. The agent with the most success links with one of any of the other agents. 


\subsubsection{Verification and Sensitivity Analysis}

Model verification can be done iteratively in the development of a model and also via parameter sweeps. When done iteratively, this is referred to as progressive debugging. A method of parameter sweeps that can be used includes extreme combination tests (An et al 2005). In order to verify the model, I used a progressive debugging method to ensure a feature worked properly before adding the next feature. Additionally, I conducted parameter sweeps to both test the sensitivity of the parameters and to examine the operation of the model when extreme parameters were used in combination.

The verification tests resulted in segregated network behavior that mirrors the segregation levels in spatial models of similar preference. Additionally, the preferential attachment produced degree distributions mirroring power law distributions of a scale free network (Barabasi and Bonabeau 2003). For the sensitivity analysis, I used Behavior Space within Netlogo to conduct a total of 720 simulations of parameter combinations in the baseline activation settings. Based on this analysis, the network building that takes place in the model is expectedly sensitive to preferential attachment behavior. In terms of the impact on the percentage of minorities, variance in results was much higher when preferential attachment was included in the network building behavior than when not, as shown in Figures 1 and 2.

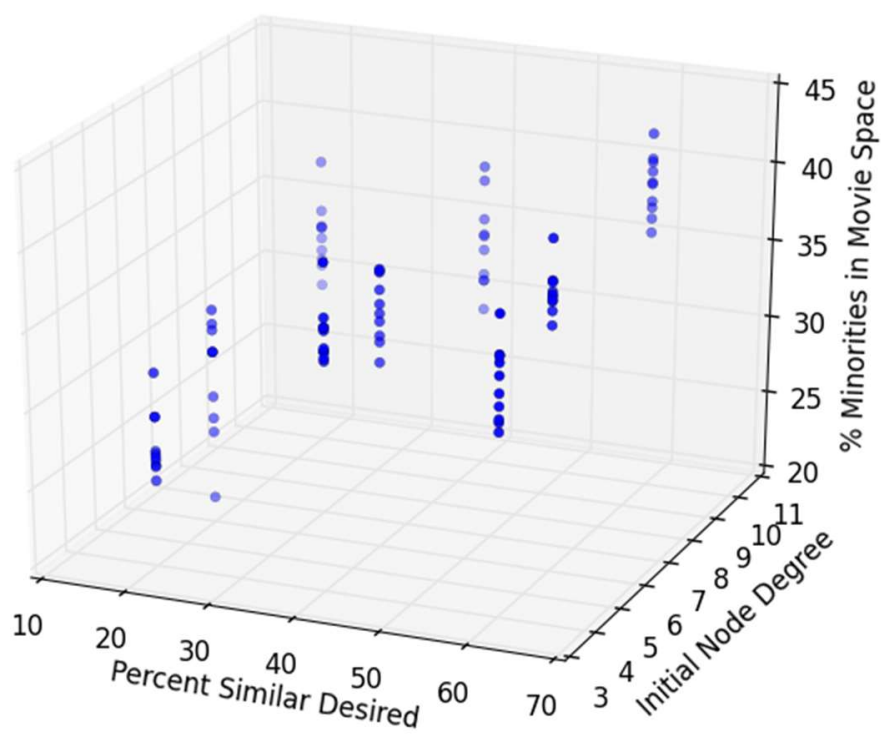

Figure 1: No Preferential Attachment. 


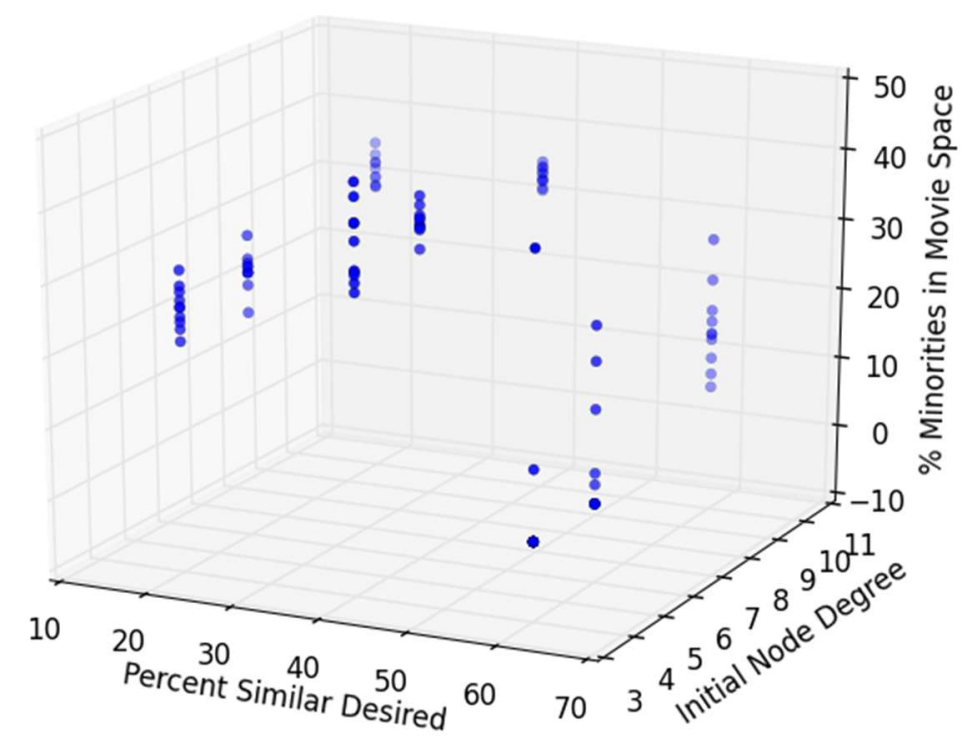

Figure 2: With Preferential Attachment.

\subsection{Social Network Analysis}

I identified a real world actor co-production network in order to explore the second hypothesis that race/ethnic minority status influences one's position within the co-production networks of successful actors. This network is also used to validate the above discussed ABM.

To construct the network of successful actors I examined the 2015 co-production networks of the top five billed actors in the top ten grossing movies of that same year. I then identified the 2015 coproduction networks for these 50 (The Numbers 2016). For each movie the ego was credited for that year, I connected them to the other credited actors of that movie. To account for the likelihood that every actor in a film did not co-perform (and thus had no real world link) to the ego in question, I capped the connected actors to the top 10 in each film. For an example of these ego networks see Figure 3. The film credit information was derived from the Integrated Movie Database (2015). 


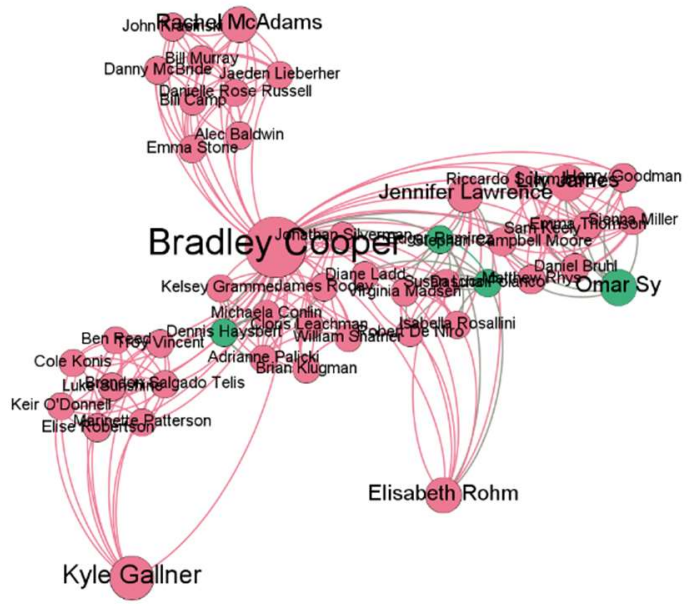

Figure 3: Example Ego Network.

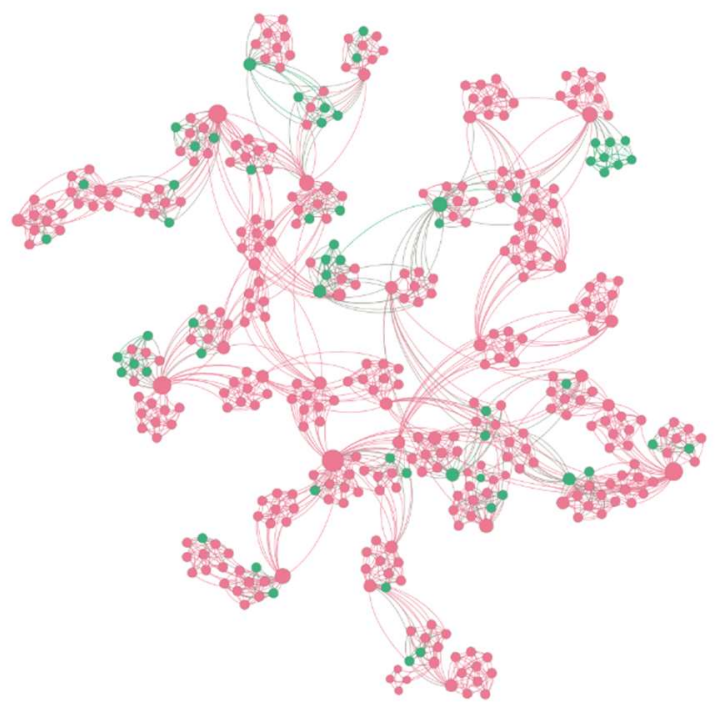

Figure 4: Giant Component of Merged Ego Networks. Size of node denotes relative degree centrality. Pink nodes denote non-minority racial identity. Green nodes denote minority racial identity.

For the social network analysis, I combined the 50 ego networks and merged links between actors. The merged ego networks create a network of co-production that consists of 406 nodes and 2033 undirected edges, as shown in Figure 4. Interestingly, the merged network consists entirely of one component, demonstrating the closed network nature of actor networks in high grossing Hollywood films.

\section{FINDINGS}

\subsection{ABM Findings and Model Validation}

Based on calibration to real world Hollywood labor market conditions and the results of the sensitivity analysis parameter sweeps, the experiments testing the hypothesis were conducted in the preferential attachment mode of the model. Based on 180 runs of the simulation, no benign level of preference mirrored the level of underrepresentation of minorities in Hollywood. Additionally, the only settings which achieved the macro level results mirroring the percentage of minorities found in Hollywood, was $60 \%$ preference for racial similarity within one's own network. However, to reach this level, the network element ceased to exist in many cases. In all of the runs, the number of links was diminished from 0 to 40 . As such, individual preference for racial and ethnic similarity within one's own network at the microlevel DOES NOT sufficiently explains the phenomena of Hollywood underrepresentation at the macrolevel.

Next, I examined what other category of hypotheses should be explored to explain the underrepresentation of minorities in Hollywood film. The two categories tested were automatic link loss and opportunity denial for minorities. Between the two categories, automatic link loss did not produce a resulting network that was usable for analysis or that replicated the phenomena. However, when the ABM was run with levels of opportunity denial for minorities added, the results approached the macro level of the Bunche Center Study's $16.7 \%$ and the 2015 top movie actor network of $14.7 \%$.

I tested 200 runs of the opportunity loss setting at levels of $50 \%, 60 \%, 70 \%$, and $80 \%$ both with and without $1 \%$ noise levels. The setting that most reliably produced the macro level results, were opportunity loss levels of $60 \%$, with a default similarity setting for all the runs of $20 \%$ with preferential attachment for higher 
status agents. Based on these findings, I would recommend testing hypotheses for denial of access to minorities with sufficient access to Hollywood networks to receive opportunities in the market.

Having explored the behavior that produces the macro level outcome of underrepresentation of minorities in Hollywood movies, I then tested for micro level behavior in the form of network analysis. Here, I compared the output of the network that accessed the movie space at the end of the simulations with the 2015 top movie actor network data using Gephi.

From a validation perspective, the model used for this experimentation is able to replicate the dynamics of the Hollywood market place at the macro level. However, further calibration and design is necessary to address whether the model is representing the dynamics at the micro level. From the perspective of the level that this model mirrors the real world, we can validate it at the macro level but not the micro level (Axtell and Epstein 1994). Additional calibration of the model should also consider whether the mapping of Hollywood networks along same movies is sufficient to capture the networks that move talent through the system.

\subsection{Social Network Analysis of 2015 Top Grossing Co-actors}

Given the agent based model findings allow us to reject the hypothesis that segregation along a network is sufficient to explain the underrepresentation of minorities in Hollywood films, we next turn to the real world network of 2015 actors and examine our second hypothesis: race/ethnic minority status influences one's position within the co-production networks of successful actors.

I first examine the network structure in Gephi. I examine degree, closeness, betweenness, and eigenvector centralities for the nodes. The descriptive statistics for these measures are in Table 1.

Next, I apply Gephi's modularity algorithm to check for distinct communities within the network. There are 14 identified communities within the network. This again is an interesting finding as it is intrinsic to the work relationships of the actors in the network rather than reflective of any number of constructed communities in the data collection process, as shown in Figure 5.

Table 1: Descriptive Statistics of Real World Data.

\begin{tabular}{|l|l|l|l|l|l|}
\hline & degree & triangles & eigenvector & closeness & betweeness \\
\hline Mean & 10.01 & 39.68 & 0.18 & 0.20 & 0.01 \\
\hline $\begin{array}{l}\text { Standard } \\
\text { Error }\end{array}$ & 0.21 & 0.86 & 0 & 0.00 & 0.00 \\
\hline Median & 9 & 36 & 0.16 & 0.20 & 0.00 \\
\hline $\begin{array}{l}\text { Standard } \\
\text { Deviation }\end{array}$ & 4.19 & 17.33 & 0.09 & 0.03 & 0.04 \\
\hline $\begin{array}{l}\text { Sample } \\
\text { Variance }\end{array}$ & 17.6 & 300.25 & 0.01 & 0.00 & 0.00 \\
\hline Minimum & 4 & 6 & 0.02 & 0.15 & 0.00 \\
\hline Maximum 46 & 189 & 1 & 0.31 & 0.47 \\
\hline
\end{tabular}




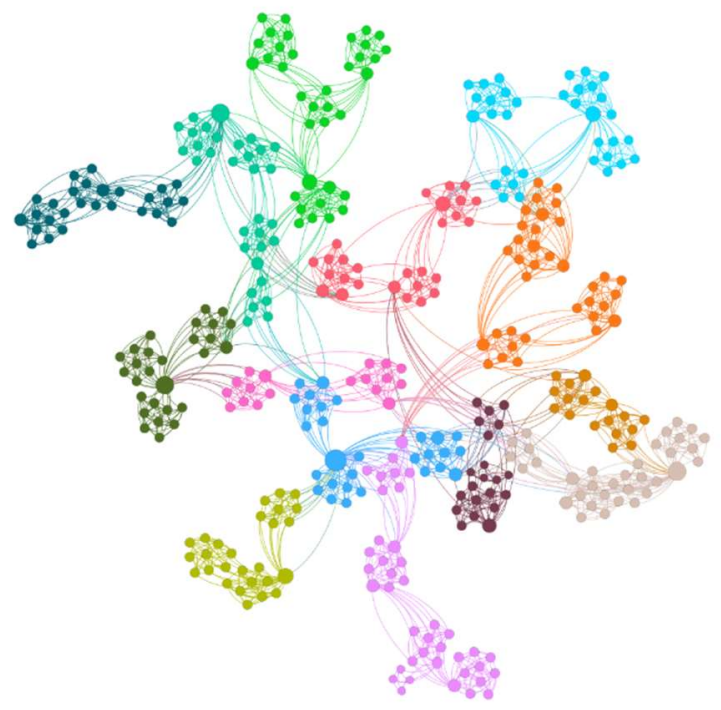

Figure 5: 2015 actor network with communities highlighted.

Given the nature of this inquiry, if race/ethnicity demonstrates an impact on network role, it should be identifiable in a linear correlation. The race variable is set as a dummy variable, setting minority to 1 . The correlation coefficients for the above centralities reveal no correlation exists.

Even if centrality is not correlated with race, there is still the possibility that race has an impact on the geodesic location of a node. Here, I use the multidimensional scaling (MDS) algorithm to identify the node's geodesic location in relation to other nodes (Algorithmics Group 2009). I then perform a regression analysis against the race dummy variable. Again, there was no relationship between race and the MDS location.

\section{CONCLUSIONS AND FUTURE WORK}

Based on a calibrated ABM of the Hollywood movie market, segregation on a network due to individual preferences for similarity is not sufficient to explain the current status of underrepresentation of minorities in Hollywood. Additionally, for individuals working in successful Hollywood films, racial/ethnic preferences are not correlated with an individual's network. Further simulation of this market and network suggests that causes of this phenomena that address lack of opportunity for minorities who already exhibit access to Hollywood networks should be explored. Structural impediments to the movie labor market should be explored to identify the nature and role of opportunity loss or denial for minority actors.

One way to test opportunity loss or denial in the real world data would be data from an anomalous year where minority populations in films were higher than previous years. While 2015 was consistent with the preceding two years, it was also demonstrative of a minor increase over previous decades. However, 2016 data for the same level of movies and their actors is a break from this consistent pattern. Minority representation in 2016 data shows an increase to 25\%. Examination of 2017-2019 data, would allow a researcher to demonstrate whether 2016 is an anomaly or the possible beginning of a trajectory shift. As such, further exploration of the phenomenon in either case merits further study. 


\section{REFERENCES}

Algorithmics Group. 2009. MDSJ: Java Library for Multidimensional Scaling (Version 0.2). http://www.inf.uni-konstanz.de/algo/software/mdsj/. University of Konstanz.

An, L., M. Linderman, J. Qi, A. Shortridge, and J. Liu. 2005. "Exploring Complexity in a HumanEnvironment System: An Agent-Based Spatial Model for Multidisciplinary and Multiscale Integration". Annals of the Association of American Geographers 95(1): 54-79.

Axtell, R and J. Epstein. 1994. "Agent-Based Modeling: Understanding Our Creations." The Bulletin Of The Santa Fe Institute, Winter 1994: 28-32.

Baker, W.E and Faulkner R.R. 1991. "Role as Resource in the Hollywood Film Industry," American Journal of Sociology, 92(2): 279-309.

Barabási, A. and E. Bonabeau. 2003. "Scale-Free Networks", Scientific American, 288(5): 50-59.

Bonilla-Silva , E. 2006. Racism without Racists: Color-Blind Racism and the Persistence of Racial Inequality in the United States, 2nd ed. Boulder, CO: Rowman and Littlefield.

Bourdieu, P., and R. Johnson. 1993. The field of cultural production : Essays on art and literature (European perspectives). New York: Columbia University Press.

Bunche Center for African American Studies at UCLA. 2016. "2016 Hollywood Diversity Report: Business as Usual”. http://www.bunchecenter.ucla.edu/wp-content/uploads/2016/02/2016-HollywoodDiversity-Report-2-25-16.pdf

DeVany, A. 2004. Hollywood Economics: How Extreme Uncertainty Shapes the Film Industry. London: Routledge.

Grimm, V., U. Berger, D. Deangelis, J. Polhill, J. Giske, and S. Railsback. 2010. "The ODD Protocol: A Review And First Update.” Ecological Modelling 221(23), 2760-2768.

Fine, G.A. and C.D. Fields. 2008. "Culture and Microsociology: The Anthill and the Veldt". The Annals of the American Academy of Political and Social Science. 619: 130-148.

Herr II, B.W., W. Ke, E. Hardy, and K. Börner. 2007. "Movies and Actors: Mapping the Internet Movie Database". In Proceedings of 11th Annual Information Visualization International Conference (IV 2007). Edited by S. Ceballos, 465-469, Piscataway, New Jersey: Institute of Electrical and Electronics Engineers, Inc..

Lam, B. 2015. "Least Diverse Jobs in America". The Atlantic. http://www.theatlantic.com/business/archive/2015/06/diversity-jobs-professions-america/396632/

Kadushin, C. 2012. Understanding Social Networks : Theories, Concepts, and Findings. New York: Oxford University Press.

Massey, D.S., N.A. Denton. 1993. American Apartheid: Segregation and the Making of the Underclass. Cambridge, MA: Harvard University Press.

Mcdonald, S. 2011. "What's in the "old boys" network? Accessing social capital in gendered and racialized networks". Social Networks. 33(4): 317-330.

McPherson, M., L. Smith-Lovin, and J.M. Cook. 2001. "Birds of a Feather: Homophily in Social Networks". Annual Review Of Sociology 27: 415-444.

Moujahid, A. 2014. "An introduction to agent-based models: simulating segregation with Python," https://www.binpress.com/tutorial/introduction-to-agentbased-models-an-implementation-ofschelling-model-in-python/144.

Rhee, M., and T. Kim. 2014. "Identity-based Learning and Segregation in Social Networks Under Different Institutional Environments". Computational and Mathematical Organization Theory 20(4): 339-368. 
Russo, H. 2016. "Explaining Box Office Performance from the Bottom Up: Data, Theory, and Models". PhD. Thesis, Department of Computational and Data Sciences, George Mason University, Fairfax, Virginia. http://mars.gmu.edu/handle/1920/10440.

Schelling, T. 1971. "Dynamic Models of Segregation". Journal of Mathematical Sociology, 1(1): 143-186.

Schelling, T. 1978. Micromotives and Macrobehavior. New York: Norton.

Smith, S., M. Choueiti, and K. Pieper. 2016. Inclusion or Invisibility: Comprehensive Annenberg Report on Diversity in Entertainment. USC Annenberg School of Communication and Journalism. http://annenberg.usc.edu/pages/ /media/MDSCI/CARDReport\%20FINAL \%2022216.ashx

Smith, Vicki ed. 2013. Sociology of Work. SAGE Publications.

Stonedahl, F. and U. Wilensky. 2008. NetLogo Virus on a Network Model. http://ccl.northwestern.edu/netlogo/models/VirusonaNetwork. Center for Connected Learning and Computer-Based Modeling, Northwestern University, Evanston, IL.

Sullivan Commission on Diversity in the Healthcare Workforce. 2004. "Missing Persons: Minorities in the Health Professions a Report by the Commission on Diversity in the Health Care Workforce". http://www.aacn.nche.edu/media-relations/SullivanReport.pdf.

US Census Bureau. 2014. https://factfinder.census.gov/faces/nav/jsf/pages/index.xhtml.

Tassier, T. 2008. "Referral Hiring and Gender Segregation in the Workplace". Eastern Economic Journal 34(4): 429.

Walls, W.D., and J. McKenzie. 2012. "The Changing Role of Hollywood in the Global Movie Market". Journal of Media Economics 25(4).

Wasserman, S., and K. Faust. 1994. Social network analysis : Methods and applications (Structural analysis in the social sciences). Cambridge, NY: Cambridge University Press.

Wilensky, U. 1997. NetLogo Segregation Model. http://ccl.northwestern.edu/netlogo/models/Segregation. Center for Connected Learning and Computer-Based Modeling, Northwestern University, Evanston, IL.

\section{ACKNOWLEDGEMENTS}

An earlier version of this paper benefitted from comments and suggestions provided by Andrew Crooks and Robert Axtell.

\section{AUTHOR BIOGRAPHY}

CARMEN IASIELLO is a PhD candidate in the Department of Computational and Data Sciences at George Mason University, Fairfax, VA. She holds an MA in East Asian Languages and Cultures from Columbia University and a BA in International Studies from American University. Her research interests include the application of agent based modeling techniques to human resources management. ciasiell@gmu.edu. 